\title{
SANDWICH-TYPE THEOREMS FOR CERTAIN INTEGRAL OPERATORS
}

\author{
H. A. AL-KHARSANI AND N. M. AL-AREEFI
}

\begin{abstract}
The purpose of the present paper is to obtain the sandwich-type theorem which contains the subordination- and superordination-preserving properties for certain integral operators defined on the space of normalized analytic functions in the open unit disk.
\end{abstract}

\section{Introduction}

Let $\mathcal{H}=\mathcal{H}(\mathbb{U})$ denote the class of functions in the open unit disk $\mathbb{U}=\{z \in \mathbb{C}:|z|<$ $1\}$. For $a \in \mathbb{C}$, let

$$
\mathcal{H}[a, n]=\left\{f \in \mathcal{H}: f(z)=a+a_{n} z^{n}+a_{n+1} z^{n+1}+\cdots\right\} .
$$

Let $f$ and $F$ be members of $\mathcal{H}$. The function $f$ is said to be subordinate to $F$, or $F$ is said to be superordinate to $f$, if there exists a function $w$ analytic in $\mathbb{U}$, with $w(0)=0$ and $|w(z)|<1$, and such that $f(z)=F(w(z))$. In such a case, we write $f \prec F$ or $f(z) \prec F(z)$. If the function $F$ is univalent in $\mathbb{U}$, then $f \prec F$ if and only if $f(0)=F(0)$ and $f(\mathbb{U}) \subset F(\mathbb{U})$ (cf. [6], [11]).

Let $\phi: \mathbb{C}^{2} \rightarrow \mathbb{C}$ and let $h$ be univalent in $\mathbb{U}$. If $p$ is analytic in $\mathbb{U}$ and satisfies the differential subordination

$$
\phi\left(p(z), z p^{\prime}(z)\right) \prec h(z) \quad(z \in \mathbb{U})
$$

then $p$ is called a solution of the differential subordination. The univalent function $q$ is called a dominant of the solutions of the differential subordination, or more simply a dominant if $p \prec q$ for all $p$ satisfying (1.2). A dominant $\tilde{q}$ that satisfies $\tilde{q} \prec q$ for all dominants $q$ of (1.2) is said to be the best dominant [6].

Let $\varphi: \mathbb{C}^{2} \rightarrow \mathbb{C}$ and let $h$ be analytic in $\mathbb{U}$. If $p$ and $\varphi\left(p(z), z p^{\prime}(z)\right)$ are univalent in $\mathbb{U}$ and satisfy the differential superordination

$$
h(z) \prec \varphi\left(p(z), z p^{\prime}(z)\right) \quad(z \in \mathbb{U}),
$$

Corresponding author: N. M. Al-Areefi.

Received May 8, 2009.

2000 Mathematics Subject Classification. 30C45.

Key words and phrases. Analytic function, differential subordinations, differential superordinations, starlike functions, convex functions. 
then $p$ is called a solution of the differential superordination. An analytic function $q$ is called a subordinant of the solutions of the differential superordination, or more simply a subordinant if $q \prec p$ for all $p$ satisfying (1.3). A univalent subordinant $\tilde{q}$ that satisfies $q \prec \tilde{q}$ for all subordinants $q$ of (1.3) is said to be the best subordinant [7].

We denote by $\mathcal{Q}$ the class of functions $f$ that are analytic and injective on $\overline{\mathbb{U}} \backslash E(f)$, where

$$
E(f)=\left\{\zeta \in \partial \mathbb{U}: \lim _{z \rightarrow \zeta} f(z)=\infty\right\},
$$

and are such that $f^{\prime}(\zeta) \neq 0$ for $\zeta \in \partial \mathbb{U} \backslash E(f)[7]$.

Let $A$ denote the subclass of $\mathcal{H}[a, 1]$ with the usual normalization $f(0)=f^{\prime}(0)-1=0$. We also denote by $\mathcal{K}(\alpha)(\alpha<1)$ the class of convex functions of order $\alpha$ in $\mathbb{U}$. That is,

$$
\mathcal{K}(\alpha):=\left\{f \in A: \operatorname{Re}\left\{1+\frac{z f^{\prime \prime}(z)}{f^{\prime}(z)}\right\}>\alpha(z \in \mathbb{U})\right\} .
$$

The class of starlike functions of order $\alpha(\alpha<1)$, denoted by $\mathcal{S}^{*}(\alpha)$, is defined by

$$
\mathcal{S}^{*}(\alpha):=\left\{f \in A: \operatorname{Re}\left\{\frac{z f^{\prime}(z)}{f(z)}\right\}>\alpha(z \in \mathbb{U})\right\} .
$$

In particular, the class $\mathcal{K} \equiv \mathcal{K}(0)$ and $\mathcal{S}^{*} \equiv \mathcal{S}^{*}(0)$, respectively, represent the classes of convex functions and starlike functions in $\mathbb{U}$.

Let denote by $\mathcal{A}$ the class

$$
\mathcal{A}=\left\{h \in A: h(z) h^{\prime}(z) \neq 0, \quad 0<|z|<1\right\} .
$$

For a function $h \in \mathcal{A}$ we define the integral operator $I_{h ; \beta}: \mathcal{K}_{h ; \beta} \rightarrow H(\mathbb{U})$ by

$$
I_{h ; \beta}[f](z)=\left[\beta \int_{0}^{z} f^{\beta}(t) h^{-1}(t) h^{\prime}(t) d t\right]^{1 / \beta}
$$

where the subset $\mathcal{K}_{h ; \beta} \subset H(\mathbb{U})$ was determined in [2], such that this integral operator is well defined (all powers are the principal ones).

In the present paper, we investigate the subordination- and superordination-preserving properties of the integral operator $I_{h ; \beta}$ defined by (1.7) with the sandwich-type theorem.

\section{Preliminaries}

The following lemmas will be required in our present investigation.

Lemma 2.1.([5]) Let $\beta, \gamma \in \mathbb{C}$ with $\beta \neq 0$ and let $h \in \mathcal{H}(\mathbb{U})$ with $h(0)=c$. If $\operatorname{Re}\{\beta h(z)+$ $\gamma\}>0(z \in \mathbb{U})$, then the solution of the differential equation

$$
q(z)+\frac{z q^{\prime}(z)}{\beta q(z)+\gamma}=h(z) \quad(z \in \mathbb{U})
$$

with $q(0)=c$ is analytic in $\mathbb{U}$ and satisfies $\operatorname{Re}\{\beta q(z)+\gamma\}>0 \quad(z \in \mathbb{U})$. 
Lemma 2.2.([6]) Let $p \in \mathcal{Q}$ with $p(0)=a$ and let $q(z)=a+a_{n} z^{n}+\cdots$ be analytic in $\mathbb{U}$ with $q(z) \not \equiv a$ and $n \geq 1$. If $q$ is not subordinate to $p$, then there exist points $z_{0}=r_{0} e^{i \theta} \in \mathbb{U}$ and $\zeta_{0} \in \partial \mathbb{U} \backslash E(f)$, for which $q\left(\mathbb{U}_{r_{0}}\right) \subset p(\mathbb{U})$,

$$
q\left(z_{0}\right)=p\left(\zeta_{0}\right), \quad z_{0} q^{\prime}\left(z_{0}\right)=m \zeta_{0} p^{\prime}\left(\zeta_{0}\right) \quad(m \geq n) .
$$

Our next lemma deals with the notion of subordination chain. A function $L(z, t)$ defined on $\mathbb{U} \times[0, \infty)$ is the subordination chain if $L(\cdot, t)$ is analytic and univalent in $\mathbb{U}$ for all $t \in[0, \infty), L(z, \cdot)$ is continuously differentiable on $[0, \infty)$ for all $z \in \mathbb{U}$, and $L(z, s) \prec L(z, t)$ for $z \in \mathbb{U}$ and $0 \leq s<t$.

Lemma 2.3.([7]) Let $q \in \mathcal{H}[a, 1]$, let $\varphi: \mathbb{C}^{2} \rightarrow \mathbb{C}$, and set $\varphi\left(q(z), z q^{\prime}(z)\right) \equiv h(z)$. If $L(z, t)=\varphi\left(q(z), t z q^{\prime}(z)\right)$ is a subordination chain and $p \in \mathcal{H}[a, 1] \cap \mathcal{Q}$, then

$$
h(z) \prec \varphi\left(p(z), z p^{\prime}(z)\right) \quad(z \in \mathbb{U})
$$

implies that

$$
q(z) \prec p(z) \quad(z \in \mathbb{U}) .
$$

Furthermore, if $\varphi\left(q(z), z p^{\prime}(z)\right)=h(z)$ has a univalent solution $q \in \mathcal{Q}$, then $q$ is the best subordinant.

We now recall that the Gauss hypergeometric function ${ }_{2} F_{1}(a, b ; c ; z)$ is defined by [9], (see also [12, Chapter 14])

$$
{ }_{2} F_{1}(a, b ; c ; z):=\sum_{n=0}^{\infty} \frac{(a)_{n}(b)_{n}}{(c)_{n}} \frac{z^{n}}{n !}\left(z \in \mathbb{U} ; b \in \mathbb{C} ; c \in \mathbb{C} \backslash \mathbb{Z}_{0}^{-} ; \mathbb{Z}_{0}^{-}:=\{0,-1,-2, \ldots\}\right),
$$

where $(\lambda)_{\nu}$, denotes the Pochhammer symbol (or the shifted factorial) defined (for $\lambda, \nu, \in \mathbb{C}$ and in terms of the Gamma function) by

$$
(\lambda)_{\nu}:=\frac{\Gamma(\lambda+\nu)}{\Gamma(\lambda)}= \begin{cases}1 & (\nu=0 ; \lambda \in \mathbb{C} \backslash\{0\}), \\ \lambda(\lambda+1) \cdots(\lambda+\nu-1) & (\nu=n \in \mathbb{N} ; \lambda \in \mathbb{C}) .\end{cases}
$$

Lemma 2.4.([8]) Let $\beta>0, \beta+\gamma>0$ and let $I_{\beta, \gamma}$ be the integral operator defined by (1.7). If $\alpha \in[-\gamma / \beta, 1)$, then the order of starlikeness of the class $I_{\beta, \gamma}\left(\mathcal{S}^{*}(\alpha)\right)$, that is, the largest number $\delta=\delta(\alpha ; \beta, \gamma)$ such that

$$
I_{\beta ; \gamma}\left(\mathcal{S}^{*}(\alpha)\right) \subset \mathcal{S}^{*}(\delta),
$$

is given by the number $\delta(\alpha ; \beta, \gamma)=\inf \{\operatorname{Re} q(z): z \in \mathbb{U}\}$, where

$$
q(z)=\frac{1}{\beta Q(z)}-\frac{\gamma}{\beta}, \quad Q(z)=\int_{0}^{1}\left(\frac{1-z}{1-t z}\right)^{2 \beta(1-\alpha)} t^{\beta+\alpha-1} d t .
$$

Moreover, if $\alpha \in\left[\alpha_{0}, 1\right)$, where

$$
\alpha_{0}:=\max \left\{\frac{\beta-\gamma-1}{2 \beta},-\frac{\gamma}{\beta}\right\}
$$


and $f \in \mathcal{S}^{*}(\alpha)$, then

$$
\operatorname{Re}\left\{\frac{z\left(I_{\beta ; \gamma}(f)(z)\right)^{\prime}}{I_{\beta ; \gamma}(f)(z)}\right\}>\delta(\alpha ; \beta, \gamma)=\frac{1}{\beta}\left[\frac{\beta+\gamma}{{ }_{2} F_{1}(1,2 \beta(1-\alpha), \beta+\gamma+1 ; 1 / 2)}-\gamma\right\},
$$

where ${ }_{2} F_{1}$ represents the Gauss hypergeometric function defined by (2.5).

Lemma 2.5.([10]) The function $L(z, t)=a_{1}(t) z+\cdots$, with $a_{1}(t) \neq 0$ and $\lim _{t \rightarrow \infty}\left|a_{1}(t)\right|=$ $\infty$, is a subordination chain if and only if

$$
\operatorname{Re}\left\{\frac{z \partial L(z, t) / \partial z}{\partial L(z, t) / \partial t}\right\}>0 \quad(z \in \mathbb{U}, 0 \leq t<\infty) .
$$

Throughout this paper, we will denote $A_{h ; \beta}$ by

$$
A_{h ; \beta}:=\left\{f \in A: \frac{f(z)}{z} \neq 0, \frac{I_{h ; \beta}(f)(z)}{z} \neq 0(z \in \mathbb{U} ; \operatorname{Re}\{\beta\}>0)\right\} .
$$

where $I_{h ; \beta}$ is the integral operator defined by (1.7).

In [1], and [2], the author determined conditions on the $h$ and $g$ functions and on the parameter $\beta$, such that

$$
\left[\frac{z h^{\prime}(z)}{h(z)}\right]^{1 / \beta} f(z) \prec\left[\frac{z h^{\prime}(z)}{h(z)}\right]^{1 / \beta} g(z) \Rightarrow I_{h ; \beta}[f](z) \prec I_{h ; \beta}[g](z),
$$

and in [2] was studied the reverse problem, in order to give simple sufficient conditions on $h, g$ and $\beta$, such that

$$
\left[\frac{z h^{\prime}(z)}{h(z)}\right]^{1 / \beta} g(z) \prec\left[\frac{z h^{\prime}(z)}{h(z)}\right]^{1 / \beta} f(z) \Rightarrow I_{h ; \beta}[g](z) \prec I_{h ; \beta}[f](z) .
$$

In [3], the author improved the result given in [2], finding two types of simple sufficient conditions on $h$ and $\beta$, such that the implication

$$
\begin{gathered}
{\left[\frac{z h^{\prime}(z)}{h(z)}\right]^{1 / \beta} g_{1}(z) \prec\left[\frac{z h^{\prime}(z)}{h(z)}\right]^{1 / \beta} f(z) \prec\left[\frac{z h^{\prime}(z)}{h(z)}\right]^{1 / \beta} g_{2}(z)} \\
\Rightarrow I_{h ; \beta}\left[g_{1}\right](z) \prec I_{h ; \beta}[f](z) \prec I_{h ; \beta}\left[g_{2}\right](z)
\end{gathered}
$$

holds for all $g_{1}$ and $g_{2} \alpha$-convex functions of a given order $\theta$.

\section{Main results}

Subordination theorem involving the integral operator $I_{h ; \beta}$ defined (1.7) is contained in Theorem 3.1 below. 
Theorem 3.1. Let $f, g \in A_{h ; \beta}$ with $0<\beta \leq 1$. Suppose that

$$
\operatorname{Re}\left\{1+\frac{z \phi^{\prime \prime}(z)}{\phi^{\prime}(z)}\right\}>-\frac{\beta}{2}\left(z \in \mathbb{U} ; \phi(z):=\frac{z h^{\prime}(z)}{h(z)}\left(\frac{g(z)}{z}\right)^{\beta}\right) .
$$

Then

$$
\frac{z h^{\prime}(z)}{h(z)}\left(\frac{f(z)}{z}\right)^{\beta} \prec \frac{z h^{\prime}(z)}{h(z)}\left(\frac{g(z)}{z}\right)^{\beta} \quad(z \in \mathbb{U})
$$

implies that

$$
\left(\frac{I_{h ; \beta}(f)(z)}{z}\right)^{\beta} \prec\left(\frac{I_{h ; \beta}(g)(z)}{z}\right)^{\beta} \quad(z \in \mathbb{U}),
$$

where the integral operator $I_{h ; \beta}$ is defined by (1.7). Moreover, the function $\left(\frac{I_{h ; \beta}(g)(z)}{z}\right)^{\beta}$ is the best dominant.

Proof. Let us define the functions $F$ and $G$ by

$$
F(z):=\left(\frac{I_{h ; \beta}(f)(z)}{z}\right)^{\beta}, G(z):=\left(\frac{I_{h ; \beta}(g)(z)}{z}\right)^{\beta},
$$

respectively. Without loss of generality, we can assume that $G$ is analytic and univalent on $\overline{\mathbb{U}}$, and $G^{\prime}(\xi) \neq 0$ for $|\xi|=1$.

We first show that if the function $q$ is defined by

$$
q(z):=1+\frac{z G^{\prime \prime}(z)}{G^{\prime}(z)} \quad(z \in \mathbb{U})
$$

then

$$
\operatorname{Re}\{q(z)\}>0 \quad(z \in \mathbb{U}) .
$$

From the definition of (1.7), we obtain

$$
\left(I_{h ; \beta} g(z)\right)^{\beta}\left(\frac{\left.I_{h ; \beta}(g)(z)\right)^{\prime}}{I_{h ; \beta}(g)(z)}\right)=\frac{g^{\beta}(z) h^{\prime}(z)}{h(z)} .
$$

We also have

$$
\beta\left[\frac{z\left(I_{h ; \beta}(g)(z)\right)^{\prime}}{I_{h ; \beta}(g)(z)}-1\right]=\frac{z G^{\prime}(z)}{G(z)} .
$$

It follows from (3.7) and (3.8) that

$$
\phi(z)=G(z)+\frac{1}{\beta} z G^{\prime}(z) .
$$

Now, by differentiating both sides of (3.9), we obtain

$$
q(z)+\frac{z q^{\prime}(z)}{q(z)+\beta}=1+\frac{z \phi^{\prime \prime}(z)}{\phi^{\prime}(z)} \equiv h(z) .
$$


From (3.1), we have

$$
\operatorname{Re}\{h(z)+\beta\}>\frac{\beta}{2}>0 \quad(z \in \mathbb{U}),
$$

and by using Lemma 2.1, we conclude that the differential equation (3.10) has a solution $q \in H(\mathbb{U})$ with $q(0)=h(0)=1$.

Next, we will use Lemma 2.4 for special case $\beta:=\bar{\beta}$ and $\gamma:=0$. It is easy to check that the conditions of this lemma are satisfied if $0 \leq \bar{\beta} \leq 1$ and

$$
\alpha=\alpha_{0}=\max \left\{\frac{\tilde{\beta}-1}{2 \tilde{\beta}}, 0\right\} \text {. }
$$

Then, it follows that

$$
\operatorname{Re}\{q(z)\}>\frac{1}{{ }_{2} F_{1}\left(1,2 \beta, \beta+1 ; \frac{1}{2}\right)} \geq 0 \quad(z \in \mathbb{U}) .
$$

That is, $G$ defined by (3.4) is convex (univalent) in $\mathbb{U}$.

Next, we prove that the subordination condition (3.2) implies that

$$
F(z) \prec G(z) \quad(z \in \mathbb{U})
$$

for the functions $F$ and $G$ defined by (3.4). For this purpose, we consider the function $L(z, t)$ given by

$$
L(z, t):=G(z)+\frac{1+t}{\beta} z G^{\prime}(z) \quad(z \in \mathbb{U} ; 0 \leq t<\infty) .
$$

We note that

$$
\left.\frac{\partial L(z, t)}{\partial z}\right|_{z=0}=\frac{\beta+1+t}{\beta} G^{\prime}(0) \neq 0 \quad(0 \leq t<\infty ; \beta>0) .
$$

This shows that the function

$$
L(z, t)=a_{1}(t) z+\cdots
$$

satisfies the condition $a_{1}(t) \neq 0$ for all $t \in[0, \infty)$. Futhermore, we have

$$
\operatorname{Re}\left\{\frac{z \partial L(z, t) / \partial z}{\partial L(z, t) / \partial t}\right\}=\operatorname{Re}\left\{\beta+(1+t)\left(1+\frac{z G^{\prime \prime}(z)}{G^{\prime}(z)}\right)\right\}>0,
$$

since $G$ is convex and $\beta>0$. Therefore, by virtue of Lemma $2.5, L(z, t)$ is a subordination chain. We observe from the definition of a subordination chain that

$$
\phi(z)=G(z)+\frac{1}{\beta} z G^{\prime}(z)=L(z, 0), L(z, 0) \prec L(z, t) \quad(z \in \mathbb{U} ; 0 \leq t<\infty) .
$$

This implies that

$$
L(\xi, t) \notin L(\mathbb{U}, 0)=\phi(\mathbb{U})
$$


for $\xi \in \partial \mathbb{U}$ and $t \in[0, \infty)$.

Now, suppose that $F$ is not subordinate to $G$. Then, by Lemma 2.2, there exist points $z_{0} \in \mathbb{U}$ and $\xi_{0} \in \partial \mathbb{U}$ such that

$$
F\left(z_{0}\right)=G\left(\xi_{0}\right), z_{0} F^{\prime}\left(z_{0}\right)=(1+t) \xi_{0} G^{\prime}\left(\xi_{0}\right) \quad(0 \leq t<\infty) .
$$

Hence, we have

$$
L\left(\xi_{0}, t\right)=G\left(\xi_{0}\right)+\frac{1+t}{\beta} \xi_{0} G^{\prime}\left(\xi_{0}\right)=F\left(z_{0}\right)+\frac{1}{\beta} z_{0} F^{\prime}\left(z_{0}\right)=\frac{z h^{\prime}\left(z_{0}\right)}{h\left(z_{0}\right)}\left(\frac{f\left(z_{0}\right)}{z_{0}}\right)^{\beta} \in \phi(\mathbb{U})
$$

by virtue of the suboradination condition (3.2). This contradicts the above observation that $L\left(\xi_{0}, t\right) \notin \phi(\mathbb{U})$. Therefore, the subordination condition (3.2) must imply the subordination given by (3.14). Considering $F(z)=G(z)$, we see that the function $G$ is the best dominant. Therefore, we complete the proof of Theorem 3.1.

We next prove a dual problem of Theorem 3.1 in the sense that the subordinations are replaced by supeordinations.

Theorem 3.2. Let Let $f, g \in A_{h ; \beta}$ with $0<\beta \leq 1$. Suppose that

$$
\operatorname{Re}\left\{1+\frac{z \phi^{\prime \prime}(z)}{\phi^{\prime}(z)}\right\}>-\frac{\beta}{2}\left(z \in \mathbb{U} ; \phi(z):=\frac{z h^{\prime}(z)}{h(z)}\left(\frac{g(z)}{z}\right)^{\beta}\right) .
$$

If $\frac{z h^{\prime}(z)}{h(z)}\left(\frac{f(z)}{z}\right)^{\beta}$ is univalent in $\mathbb{U}$ and $\left(\frac{I_{h ; \beta}(f)(z)}{z}\right)^{\beta} \in \mathcal{Q}$, then

$$
\frac{z h^{\prime}(z)}{h(z)}\left(\frac{g(z)}{z}\right)^{\beta} \prec \frac{z h^{\prime}(z)}{h(z)}\left(\frac{f(z)}{z}\right)^{\beta} \quad(z \in \mathbb{U})
$$

implies that

$$
\left(\frac{I_{h ; \beta}(g)(z)}{z}\right)^{\beta} \prec\left(\frac{I_{h ; \beta}(f)(z)}{z}\right)^{\beta} \quad(z \in \mathbb{U}),
$$

where that integral operator $I_{h ; \beta}$ is defined by (1.7). Moreover, the function $\left(I_{h ; \beta}(g)(z) / z\right)^{\beta}$ is the best subordinant

Proof. The first part of the proof is similar to that of Theorem 3.1 and so we will use the same notation as in the proof of Theorem 3.1.

Now, let us define the functions $F$ and $G$ respectively, by (3.4). We first note that from (3.7) and (3.8), we obtain

$$
\phi(z)=G(z)+\frac{1}{\beta} z G^{\prime}(z)=: \varphi\left(G(z), z G^{\prime}(z)\right) .
$$


After a simple calculation, (3.26) yields the following relationship

$$
1+\frac{z \phi^{\prime \prime}(z)}{\phi^{\prime}(z)}=q(z)+\frac{z q^{\prime}(z)}{q(z)+\beta},
$$

where the function $q$ is defined by (3.5). Then, by using the same method as in the proof of Theorem 3.1, we can prove that $\operatorname{Re}\{q(z)\}>0$ for all $z \in \mathbb{U}$. That is, $G$ defined by (3.4) is convex (univalent) in $\mathbb{U}$.

Next, we prove that the subordination condition (3.24) implies that

$$
F(z) \prec G(z) \quad(z \in \mathbb{U})
$$

for the functions $F$ and $G$ defined by (3.4). Now consider the function $L(z, t)$ defined by

$$
L(z, t):=G(z)+\frac{t}{\beta} z G^{\prime}(z) \quad(z \in \mathbb{U} ; 0 \leq t<\infty) .
$$

Since $G$ is convex and $\beta>0$, we can easily prove that $L(z, t)$ is a subrodination chain as in the proof of Theorem 3.1. Therefore, according to Lemma 2.3, we conclude that the superordination condition (3.24) must imply the superordination given by (3.28) Furthermore, since the differential equation (3.26) has the univalent solution $G$, it is the best subordinant of the given differential superordination. Therefore, we complete the proof of Theorem 3.2 .

If we combine Theorems 3.1 and 3.2 , then we obtain the following sandwich type theorem;

Theorem 3.3. Let $f, g_{k} \in A_{h ; \beta}(k=1,2)$ with $0<\beta \leq 1$. Suppose that

$$
\operatorname{Re}\left\{1+\frac{z \phi_{k}^{\prime \prime}(z)}{\phi_{k}^{\prime}(z)}\right\}>-\frac{\beta}{2}\left(z \in \mathbb{U} ; \phi_{k}(z):=\frac{z h^{\prime}(z)}{h(z)}\left(\frac{g_{k}(z)}{z}\right)^{\beta} ; \quad k=1,2\right) \text {. }
$$

If $\frac{z h^{\prime}}{h}\left(\frac{f(z)}{z}\right)^{\beta}$ is univalent in $\mathbb{U}$ and $\left(I_{h ; \beta}(f)(z) / z\right)^{\beta} \in \mathcal{Q}$, then

$$
\frac{z h^{\prime}(z)}{h(z)}\left(\frac{g_{1}(z)}{z}\right)^{\beta} \prec \frac{z h^{\prime}(z)}{h(z)}\left(\frac{f(z)}{z}\right)^{\beta} \prec \frac{z h^{\prime}(z)}{h(z)}\left(\frac{g_{2}(z)}{z}\right)^{\beta} \quad(z \in \mathbb{U})
$$

implies that

$$
\left(\frac{I_{h ; \beta}\left(g_{1}\right)(z)}{z}\right)^{\beta} \prec\left(\frac{I_{h ; \beta}(f)(z)}{z}\right)^{\beta} \prec\left(\frac{I_{h ; \beta}\left(g_{2}\right)(z)}{z}\right)^{\beta} \quad(z \in \mathbb{U}),
$$

where $I_{h ; \beta}$ is the integral operator digined by (1.7). Moreover, the functions $\left(I_{h ; \beta}\left(g_{1}\right)(z) / z\right)^{B}$ and $\left(I_{h ; \beta}\left(g_{2}\right)(z) / z\right)^{\beta}$ are the best subordinant and the best dominant, respectively. 
Since the assumption of Theorem 3.3 that the funcion $\left(z h^{\prime} / h\right)(f(z) / z)^{\beta}$ and $\left.I_{h ; \beta}(f)(z) / z\right)^{\beta}$ need to be univalent in $\mathbb{U}$,is not so easy to check, we will replace these conditions by another conditions in the following result.

Corollary 3.4. Let $f, g_{k} \in A_{h ; \beta}(k=1,2)$ with $0<\beta \leq 1$. Suppose that the condition (3.30) is satisfied and

$$
\operatorname{Re}\left\{1+\frac{z \psi^{\prime \prime}(z)}{\psi^{\prime}(z)}\right\}>-\frac{\beta}{2} \quad\left(z \in \mathbb{U} ; \psi(z):=\frac{z h^{\prime}(z)}{h(z)}\left(\frac{f(z)}{z}\right)^{\beta} ; \quad f \in \mathcal{Q}\right) .
$$

Then

$$
\frac{z h^{\prime}(z)}{h(z)}\left(\frac{g_{1}(z)}{z}\right)^{\beta} \prec \frac{z h^{\prime}(z)}{h(z)}\left(\frac{f(z)}{z}\right)^{\beta} \prec \frac{z h^{\prime}(z)}{h(z)}\left(\frac{g_{2}(z)}{z}\right)^{\beta} \quad(z \in \mathbb{U})
$$

implies that

$$
\left(\frac{I_{h ; \beta}\left(g_{1}\right)(z)}{z}\right)^{\beta} \prec\left(\frac{I_{h ; \beta}(f)(z)}{z}\right)^{\beta} \prec\left(\frac{I_{h ; \beta}\left(g_{2}\right)(z)}{z}\right)^{\beta} \quad(z \in \mathbb{U}),
$$

where $I_{h ; \beta}$ is the integral operator defined by (1.7). Moreover, the functions $\left(I_{h ; \beta}\left(g_{1}\right)(z) / z\right)^{\beta}$ and $\left(I_{h ; \beta}\left(g_{2}\right)(z) / z\right)^{\beta}$ are the best subordinant and the best dominant, respectively.

Proof. In order to prove Corollary 3.4, we have to show that the condition (3.33) implies the univalence of $\psi(z)$ and $F(z):=\left(I_{h ; \beta}(f)(z) / z\right)^{\beta}$. Since the conditon (3.33) means that $\psi$ is a close-to-convex function in $\mathbb{U}$ (see [4]), it follows that $\psi$ is univalent in $\mathbb{U}$. Furthermore, by using the same technigues as in the proof of Theorem 3.1, we can prove the convexity (univalence) of $F$ and so the details may be omitted. Therefore, from Theorem 3.3, we obtain Corollary 3.4.

\section{References}

[1] T. Bulboaca, On a special integral the subordination, Indian Journal of Pure and Applied Mathematics, 28(3) (1997), 361-369.

[2] T. Bulboaca, Sandwich-type theroems for a class of integral operators, The Bulletin of the Belgian Mathematical Society-Simon Stevin, 13(3) (2006), 537-550.

[3] T. Bulboaca, A class of double subordination-preserving integral operators, Pure Mathematics and Applications, 15(2-3) (2004), 87-106.

[4] W. Kaplan, Close-to-convex Schlicht functions, The Michigan Mathematical Journal, 1(2) (1952), 169-185.

[5] S. S. Miller and P.T. Mocanu, Univlaent solutions of Briot-Bouquet differntial equations, Journal of Differential Equations, 56(3) (1985), 297-309.

[6] S. S. Miller and P.T. Mocanu, Differantial Subordinations, Theory and Applications, Monographs and Textbook in Pure and Applied Mathematics, Marcel Dekker, New York, NY, USA, 225(2000). 
[7] S. S. Miller and P.T. Mocanu, Subordinants of differential supeordinations, Complex Variables. Theory and Application, 48(10) (2003), 815-826.

[8] P. T. Mocanu, D. Ripeanu, and I. Serb, The order of starlikeness of certain integral operators, Mathematica (Cluj), 23(46)(2) (1981), 225-230.

[9] S. Owa and H. M. Srivastava, Univalent and starlike generalized hypergeometric function, Canadian Journal of Mathematics, 39(5) (1987), 1057-1077.

[10] C. Pommerenke, Univalent Functions, Vandenhoeck \& Ruprecht Gottingen, Germany, 1975.

[11] H.M. Srivastava and S. Owa, Eds, Current Topics In Analytic Function Theory, World Scientific, River Edge, NJ, USA, 1992.

[12] E. T. Whittaker and G. N. Waston, A Course of Modern Analysis, An Introducion to the General Therory of Infinite Processes and of Analytic Functions, With an Account of the Principal Transcendental Functions, Cambridge University Press, Cambridge, UK, 4th edition, 1927.

Department of Mathematics, Faculty of Science, P.O. Box 838, Dammam 31113, Kingdom of Saudi Arabia.

E-mail: hakh73@hotmail.com

Department of Mathematics, Faculty of Science, P.O. Box 838, Dammam 31113, Kingdom of Saudi Arabia.

E-mail: najarifi@hotmail.com 